Nataša Soldat

Radivoje Mitrović

Ivana Atanasovska

Radoslav Tomović

https://doi.org/10.21278/TOF.44102

ISSN 1333-1124

eISSN 1849-1391

\title{
A METHODOLOGY FOR ANALYZING RADIAL BALL BEARING VIBRATIONS
}

\begin{abstract}
Summary
This paper presents the development of a methodology for calculating radial ball bearing vibrations. First, analytical and numerical models were developed and then, the obtained results were experimentally verified. In the experiment, a test rig for a dynamic testing of bearings subjected to an external load was used. Special attention was paid to the analysis of the total bearing deformations and stiffness, being the main parameters that define the dynamic behaviour of a bearing. In order to determine total deformations of a ball bearing as an assembly, a finite element model of the bearing was developed. The results obtained from the new methodology for a particular ball bearing type have been verified by experimental results. The obtained correlations of the experimental and the numerical results are about $2.5 \%$. Based on the calculations and the experimental testing of the chosen ball bearing type, under conditions of a variable radial load, the analysis of the influence of the radial load on the dynamic behaviour of a ball bearing was performed. The analysis of the obtained results showed that the vibration amplitude increases with a similar multiple factor as loads increase.
\end{abstract}

Key words: $\quad$ radial ball bearings, dynamics, vibrations, finite element analysis

\section{Introduction}

Bearings are machine elements that enable relative motion of moving parts with a simultaneous transfer of load between them while ensuring the accuracy of their position. Due to the specific design and functioning, vibrations in bearings are inevitable, even in the cases of an ideal bearing geometry. In recent years, a great number of papers dealing with basic causes of vibrations in ball bearings have been published.

Clearance is targeted as one of the main factors influencing ball bearing vibrations. Harsha [1] developed a mathematical model for the analysis of bearing vibrations caused by excessive clearance. This model was defined for the case of the tangential movement of rolling elements, as well as inner and outer rings, using non-linear sources, such as the Hertzian contact stress and internal radial clearance. Changqing and Qingyu [2] introduced a general dynamic model for the study of dynamic characteristics of a rotor system, held in 
place by ball bearings, under the influence of the internal clearance and waviness of the outer ring. In this study, a mathematical model was used for the study of the effect of clearance, waviness, preloading and radial forces on non-linear stability as well as for the study of rotor system vibrations at high speeds. On the other hand, Tomović et al. [3] pointed out that the specific design of a bearing has a significant influence on its dynamic behaviour. In their study, they defined a new model of vibrations of a rigid rotor supported by ball bearings. The use of the model defined in this way has enabled us to obtain a better insight into the influence of internal radial clearance and the total number of rolling elements. The vibration model was also confirmed experimentally.

Besides clearance, many researchers have studied the influence of deviations of the race geometry from the ideal geometry on the dynamic behaviour of ball bearings and the vibrations $[4,5,6]$. Jang and Jeong [6] also studied the effect of waviness of contact surfaces on the dynamic behaviour of bearings. They defined a model with five degrees of freedom for studying the effect of waviness on bearing vibrations, taking also the effect of centrifugal force and gyroscopic moment on rolling elements into account. Adamczak and Zmarzły [7] analysed the relationships between the waviness parameters and the level of vibration. The waviness of bearing races was measured at the actual points of contact between balls and races. The bearing vibration was analysed in three bandwidths of frequency: low $(50-300$ $\mathrm{Hz}$ ), medium $(300-1800 \mathrm{~Hz})$ and high $(1800-10000 \mathrm{~Hz})$. The test results show that an increase in the surface waviness on inner and outer raceways causes an increase in the vibration level, especially in the case of the medium frequency bandwidth. Aktuuk [8] studied the effect of waviness of bearing races on shaft vibrations. For this purpose, he developed a dynamic model with three degrees of freedom for the simulation of radial and axial vibrations of a rigid shaft assembled with ball bearings.

Mitrovic [9] studied the influence of the load distribution on the operating characteristics of ball bearings: radial stiffness, static load and lifetime. He concluded that stiffness of the bearing is not constant, which is in line with DIN and ISO standards. It depends on the total number of rolling elements, internal radial clearance and deviation of the shape of the raceway.

Several other researchers have also considered variable stiffness as the main cause of noise and vibrations in ball bearings. Variable stiffness is a result of the fact that motion is being transmitted by a finite number of rolling elements inside ball bearings. The number of rolling elements and their positions change in the load zone with the rotation of the bearing, resulting in a periodical variation of total stiffness of the bearing assembly. This variable stiffness further produces vibrations [10, 11].

Marsh and Yantek [12] concluded that the stiffness of ball bearings consisted of four components (stiffness of the rings, rolling bodies and the cage), based on the frequency response function. Similarly, Zhang et al. [13] developed an efficient method for the determination of the coefficient of stiffness of plain bearings with hydrodynamic lubrication. A relationship was obtained between the coefficients of stiffness and the bearing load characterized by different radial clearances, different length to diameter ratios and different rotation speeds.

For better understanding of the effect of different operation parameters on vibration generation in ball bearings, it has become necessary to develop a general methodology, suitable for numerous simple applications in the analysis of changes in the dynamic behaviour of bearings with variation of these parameters. Therefore, in order to investigate the dynamic 
behaviour of the radial ball bearing as a part of a complex mechanical system, a new methodology was developed and explained in the papers by Atanasovska, Mitrović and Soldat [14-18]. The application of the developed methodology upon the investigation of the influence of external load on bearing vibrations is discussed further in this paper.

In Section 2, the bearing assembly is seen as a system of masses with elastic connections with contacts between rolling elements and races viewed as a non-linear spring. An analytical calculation of the effective coefficient of stiffness according to the Hertzian theory is presented in the text.

In Section 3, a new methodology for the calculation of ball bearing vibrations is developed. The model of radial ball bearing vibration with two degrees of freedom is reduced to the one degree of freedom model, so that the application of appropriate numerical methods for solving a nonlinear problem would be possible. In this Section, the finite element analysis is used for calculating the deformation and stress states of bearing parts.

In Section 4, based on the values of total deformation of the bearing assembly, radial stiffness is calculated, and the vibrational response of the bearing assembly is obtained and discussed.

In Section 5, the new methodology developed for analysing the dynamic behaviour of the bearing is verified experimentally.

\section{Theoretical considerations}

Elastic deformations appear when rolling elements and rolling races are in contact when subjected to a load. The action of inertia forces on the rolling elements is transferred to the cage. Due to the action of these forces on the cage, the rolling elements do not keep even distances, which affects the dynamic behaviour of the bearing and the assembly the bearing is mounted on. One of the main causes of the periodic change in bearing stiffness is the periodic change in the intensity and the direction of contact forces and contact deformations between rolling elements and roller races. This is considered as the main cause of vibrations with variable parameters.

The total deformation in the point of contact of the rolling element and the ring of the bearing, according to the Hertzian elastic contact theory, can be calculated according to the following equation [4]:

$$
\delta_{1}=\left(\frac{F_{1}}{K}\right)^{\frac{1}{n}}
$$

where: $F_{1}$ - the normal load of the rolling element, $K$ - the effective coefficient of stiffness due to the Hertzian contact effect, $n$ - the exponent depending on bearing type $(3 / 2$ for ball bearings, i.e., 10/9 for roller bearings).

Stiffness of mechanical elements is defined as the ability to resist elastic deformations under the influence of external load. In bearings, it can be expressed as the ratio of the change of value of external load to the unit relative movement of the bearing rings due to contact deformations in the direction of the action of external load.

The effective coefficient of stiffness can also be calculated according to the Hertzian theory of elastic contact deformations using the following equation: 


$$
K=\frac{1}{\left(\frac{1}{K_{i}^{1 / n}}+\frac{1}{K_{o}^{1 / n}}\right)^{n}}
$$

where: $K_{i}$ - the stiffness coefficient for the contact between the rolling element and the inner race of the bearing, $K_{o}$ - the stiffness coefficient for the contact between the rolling element and the outer race of the bearing.

Thus, for the case of a ball bearing with $n=3 / 2$, the following stands:

$$
K=\frac{1}{\left(\frac{1}{K_{i}^{\frac{2}{3}}}+\frac{1}{K_{o}^{\frac{2}{3}}}\right)^{\frac{3}{2}}}
$$

Ball bearing as an assembly represents a mechanical system characterized by three basic physical values: inertia, stiffness and damping. In the modelling of mechanical systems, inertia is represented by mass $m$, stiffness is simulated by a spring with constant stiffness $c$, while damping is simulated by a damper with a damping coefficient $d$. Action of a constant force $F$ on mass $m$ leads to the movement of mass with constant acceleration $a$.

Bearing assembly can be viewed as a system of masses with elastic connections, where the outer race is fixed inside the casing, while the inner race is strongly tied to the shaft [19].

The contacts between rolling elements and races are viewed as a non-linear spring that acts only under compression. That is, the spring under compression simulates the contact deformation as well as the resultant force, Figure 1.

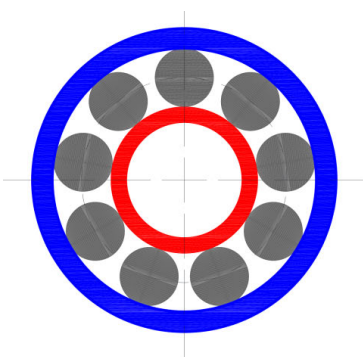

a)

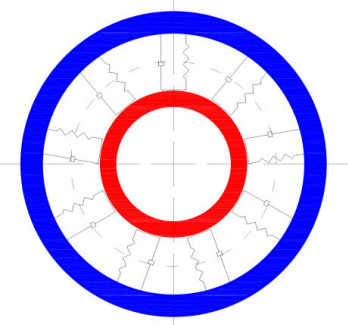

b)

Fig. 1 Contact between rolling elements and races: a) real model and b) simulation model

Elastic forces appear due to elastic deformations in the contact between rolling elements and rings of the bearing. Their intensity is time-dependent on the position of rolling elements on rolling races. Contact forces appear only in the presence of contact deformations, i.e., when the actual length of the spring is shorter than its length in an unloaded state. In the case when rolling elements and rolling races are separated, the contact force is equal to zero. In general, the time-dependent variability of intensity of contact forces is considered to be one of the most significant sources of vibrations in ball bearings. 


\section{The methodology for studying ball bearing vibrations}

In order to investigate the dynamic behaviour of the radial ball bearing as a part of a complex mechanical system, a new methodology has been developed. The main goals during the development of the methodology for the calculation of ball bearing vibrations (MCBV) were to propose a new simplified procedure based on the contemporary numerical methods which will be suitable for a wide investigation into different factors that have influence on ball bearing vibrations. The algorithm of the developed methodology is shown in Figure 2 .

\subsection{The analytical procedure for vibration calculations}

The model for the simulation of ball bearing vibrations can be explained as a deformable shaft with mass $m_{s}$ and a deformable casing with mass $m_{c}$ interconnected with the ball bearing and characterized by stiffness $c_{b}$ and damping $d_{b}$. The system of differential equations describing the vibrations of the bearing assembly in the defined model can be expressed as [16]:

$$
\mathbf{M} \ddot{\mathbf{q}}(t)+\mathbf{D} \dot{\mathbf{q}}(t)+\mathbf{C} \mathbf{q}(t)=\mathbf{F}(t)
$$

where: $\mathbf{M}$ - the mass matrix, $\mathbf{D}$ - the damping matrix, $\mathbf{C}$ - the stiffness matrix, $\mathbf{q}(\mathrm{t})$ and $\mathbf{F}(\mathrm{t})$ are vectors of the generalized movement and external forces; in the case of a radial ball bearing this vector is the time-variable radial load applied to the shaft.

The system with several degrees of freedom of movement defined in this way can be further reduced to a model of a rigid body with two degrees of freedom of movement in the case of radial bearings which transfer load only in the radial direction, Figure 3a. The analytical equations used to determine the non-linear response of vibrations then take the following form [16]:

$$
\begin{aligned}
& m_{c} \ddot{x}_{c}+d_{b}(t)\left(\dot{x}_{c}-\dot{x}_{s}\right)+c_{b}(t)\left(x_{c}-x_{s}\right)=F(t) \\
& m_{s} \ddot{x}_{s}-d_{b}(t)\left(\dot{x}_{c}-\dot{x}_{s}\right)-c_{b}(t)\left(x_{c}-x_{s}\right)=-F(t)
\end{aligned}
$$

This model can be further reduced to a system with one degree of freedom, Figure $3 \mathrm{~b}$, [16], using the reduced mass of the shaft and the casing, thus becoming the case of a deformable shaft and a rigid casing.

$$
m_{\text {red }} \ddot{x}+d_{b}(t) \dot{x}+c_{b}(t) x=F(t)
$$




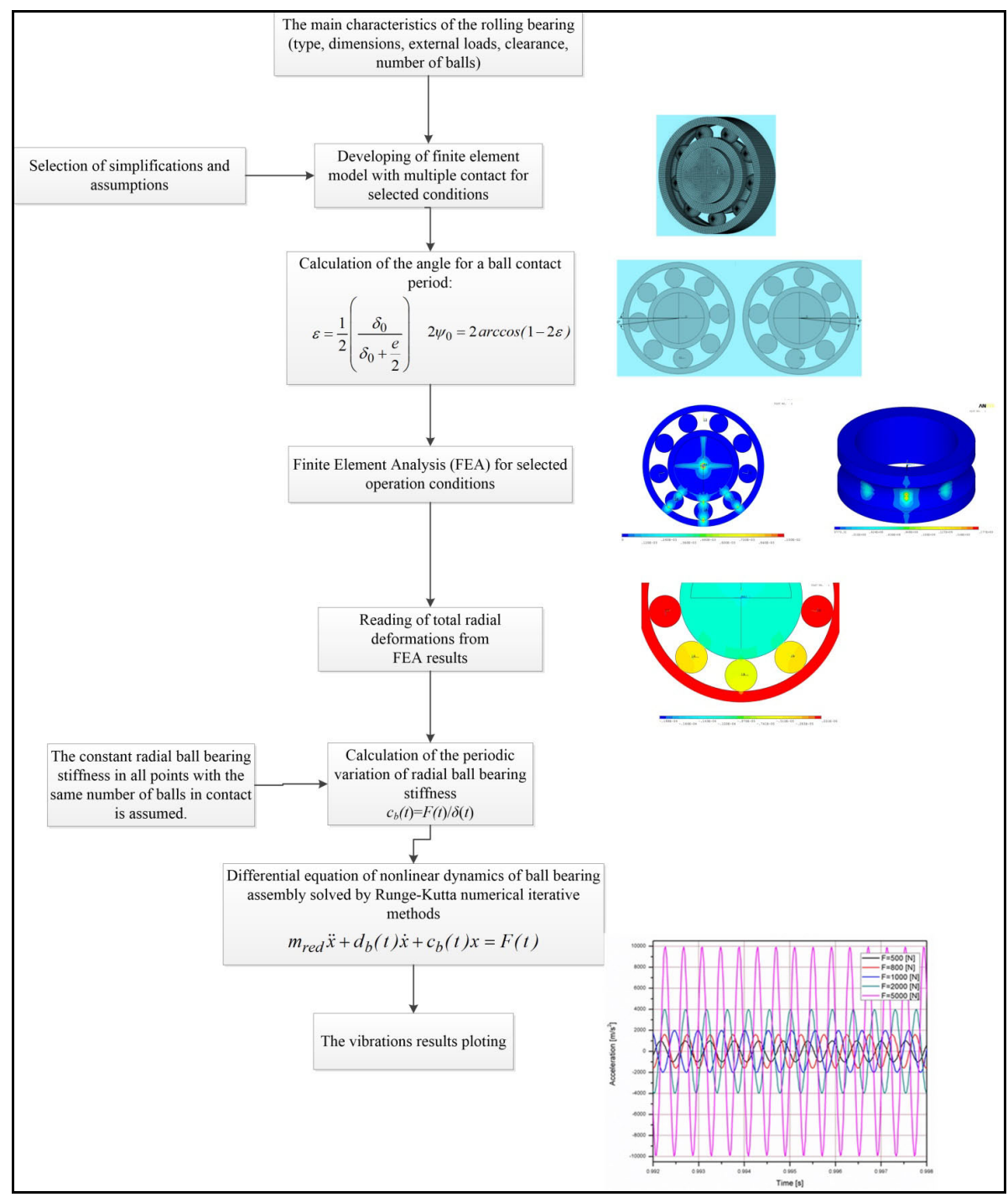

Fig. 2 Algorithm of the developed methodology

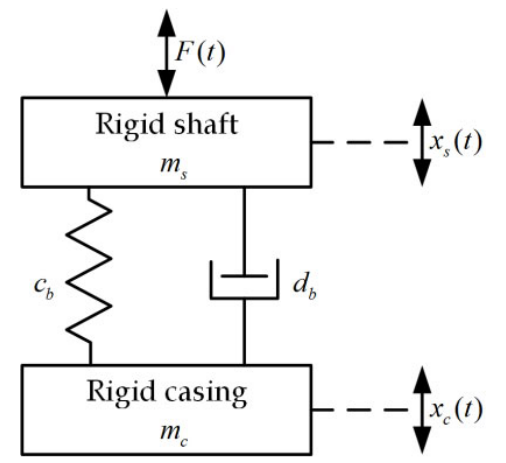

a)

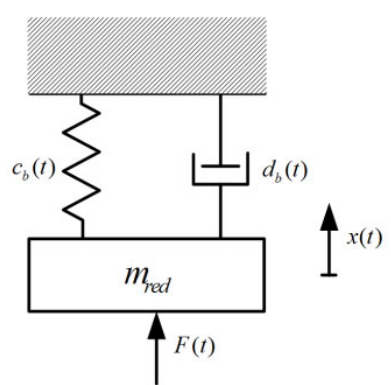

b)

Fig. 3 Model with DOF=2 (a) and single DOF reduced model (b), [16]

\subsection{The finite element model of ball bearing}

The functions required for describing the change in radial stiffness $c_{b}$ in equation (7) are determined by the total radial deformation of the bearing calculated by applying the finite element analysis (FEA) to the spatial bearing model especially developed for the cases such as this one. Software package ANSYS 13.0 and the penalty method for contact problems were used for determining the deformations and bearing stress state of the bearing parts. 
Figure 4 shows a developed model of a single-row radial ball bearing, type SKF 6206, without internal radial clearance. The bearing model consists of an outer race, an inner race, nine rolling elements and a shaft, whose diameter is equal to the diameter of the inner race of the bearing [15].

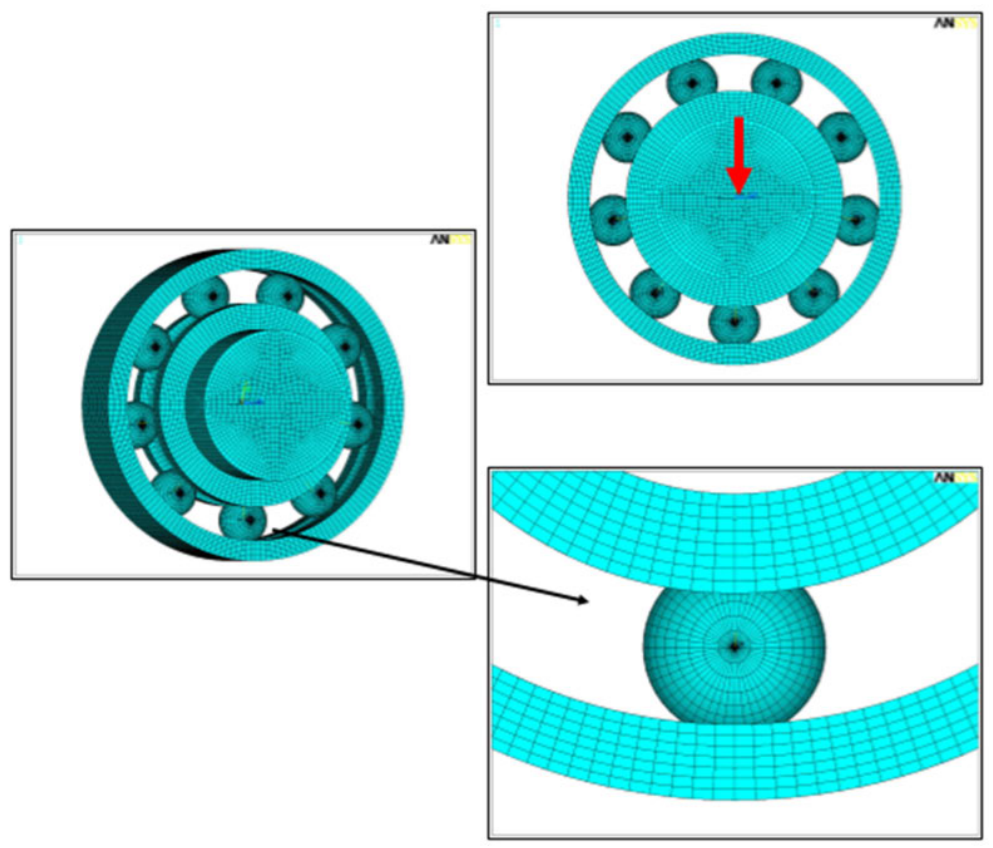

Fig. 4 3D finite element model of a rolling ball bearing

The outer race of the bearing is fixed. The inner race is limited by its contact with the shaft. The contacts are defined between the contact elements and the outer and the inner race. Boundary conditions are set in such a way that a cage with balls under certain realistic conditions is simulated. Total external load is given in the radial direction in the centre of the shaft. The following characteristics have been adopted for the bearing material is made of: module of elasticity $E=2.06 \cdot 10^{11} \mathrm{~Pa}$ and Poisson coefficient $v=0.3$.

Numerical calculations performed using the finite element analysis were used to obtain results for stress and deformation states for several different cases of external load varying from 500 to $5000 \mathrm{~N}$. For example, in this paper, the results in the case of the external radial load of $1000 \mathrm{~N}$ are presented in Figures 5 and 6. Figure 5 shows fields of equivalent VonMises stresses in two characteristic cross sections of the solid model. For other cases of external load, the contour plots for the stress and deformation results are of similar distribution. All of the finite element model results are incorporated in the presented methodology and the comparative results for different external loads are discussed below.

By analysing the contour distributions and the values of the stress state it can be concluded that the obtained results are in the expected range, considering the static load capacity of a bearing given in the manufacturer's documentation and the known characteristics of the material. Figure 6 shows the results for the total deformation in the cross section of the bearing as well as the fields of deformation of the mostly stressed bearing ball. Similar results were obtained for other cases of the simulated loads. 


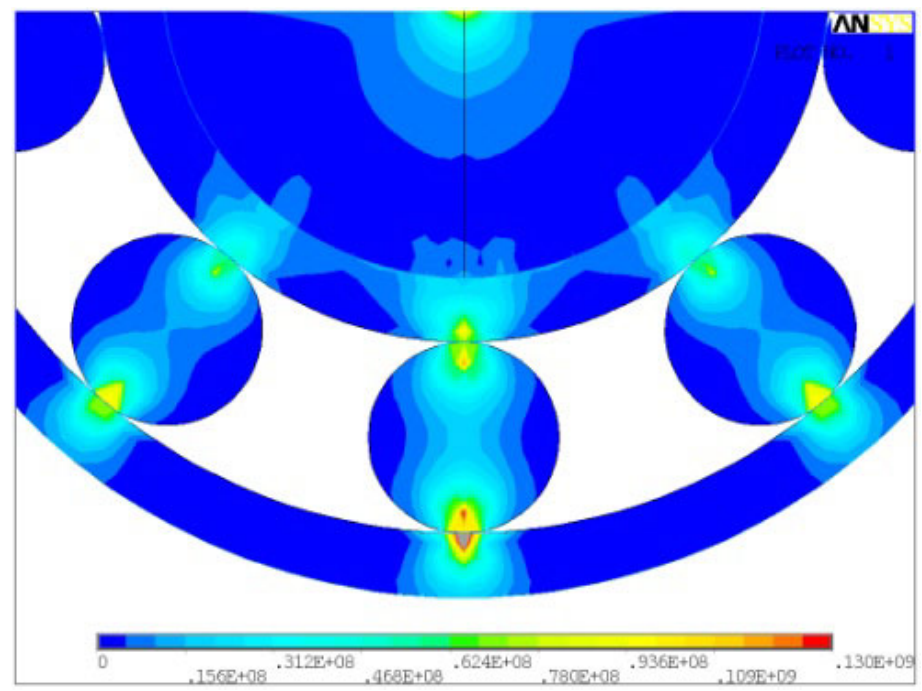

a)

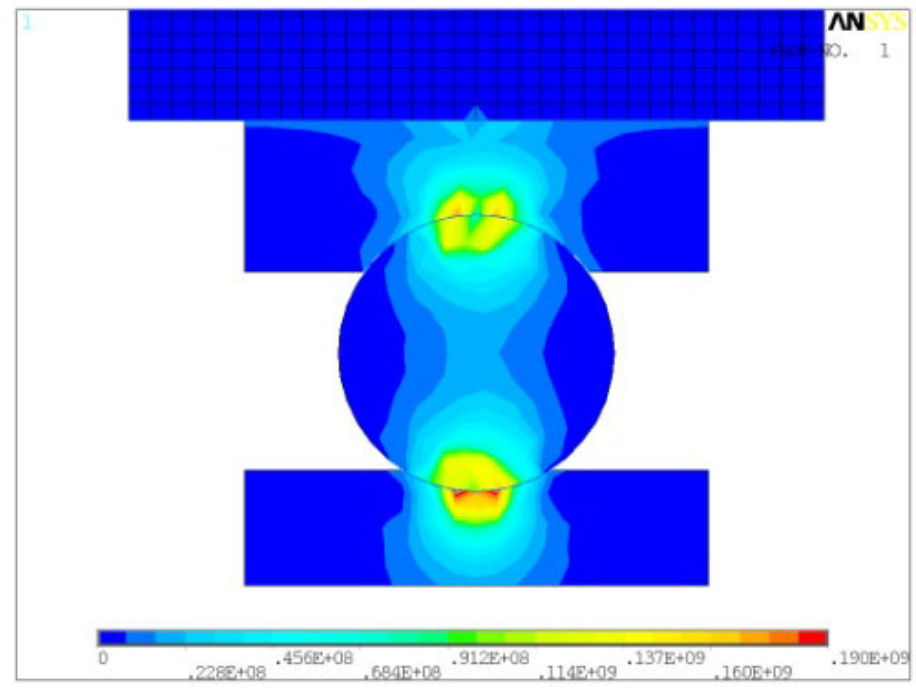

b)

Fig. 5 FEA results for equivalent VonMises stresses for 6206 bearing and load of $1000 \mathrm{~N}$ : a) face cross section of the bearing and $b$ ) side cross section of the bearing ball in contact with bearing races 


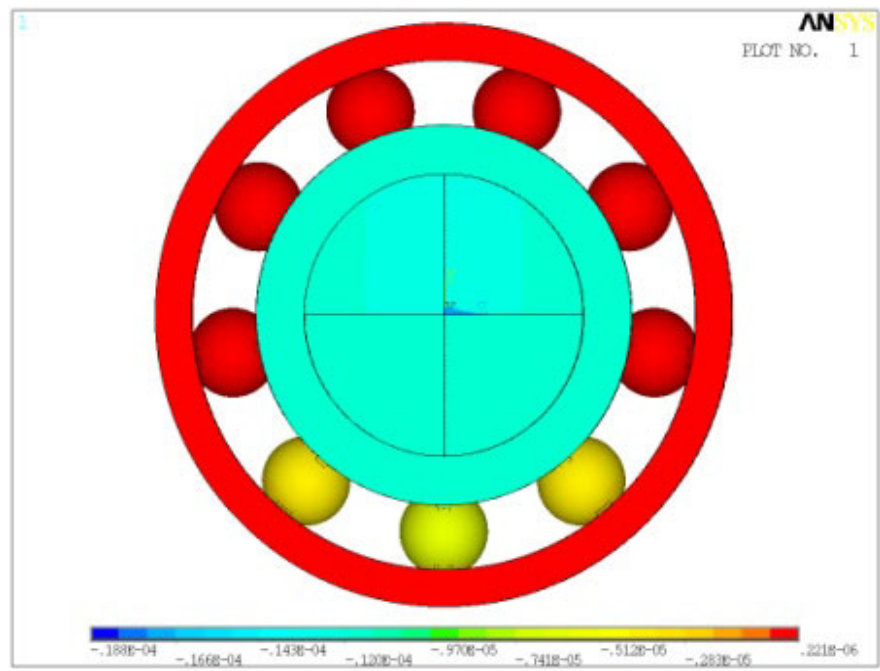

a)

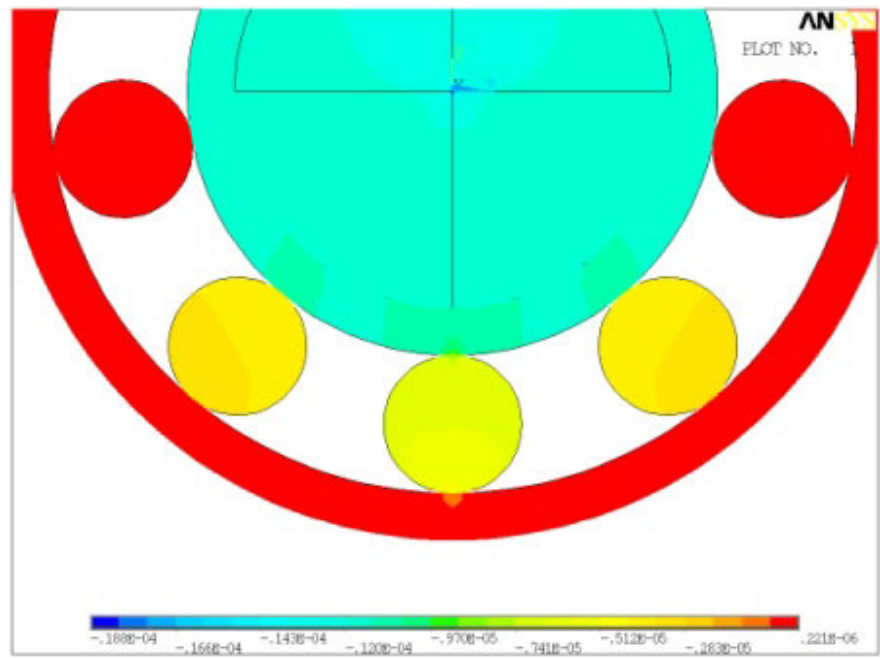

b)

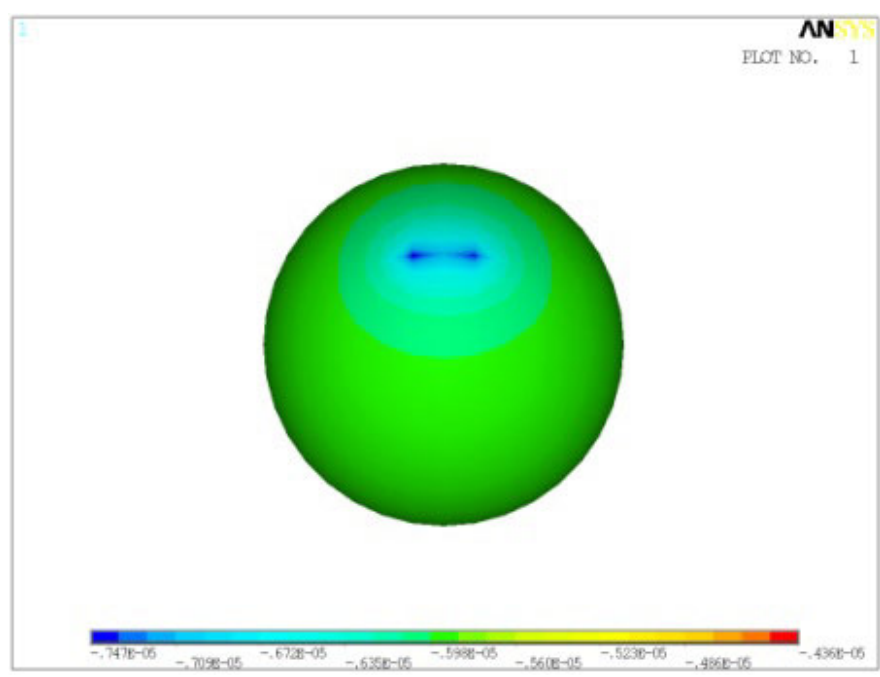

c)

Fig. 6 FEA results for displacement in radial direction for 6206 bearing for load of $1000 \mathrm{~N}$ : a) cross section of the bearing, b) zoom-in cross section of the bearing and c) rolling ball 


\section{Results and discussion}

The radial stiffness of the bearing was calculated based on the obtained values of the total deformation of the bearing as an assembly. The obtained results for the total radial deformation and radial stiffness as a function of external load for 6206 bearing are shown in Figure 7.

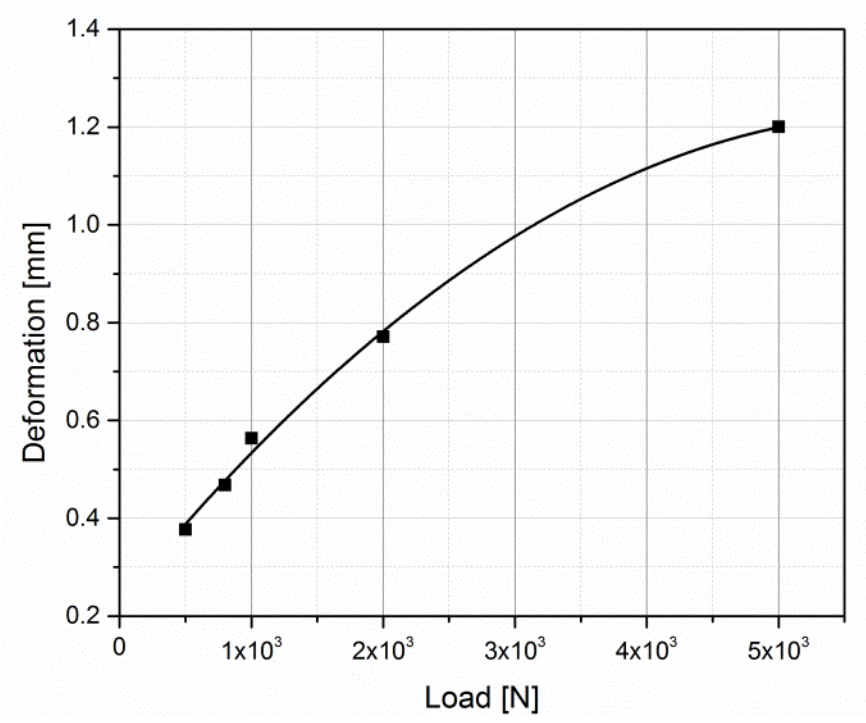

a)

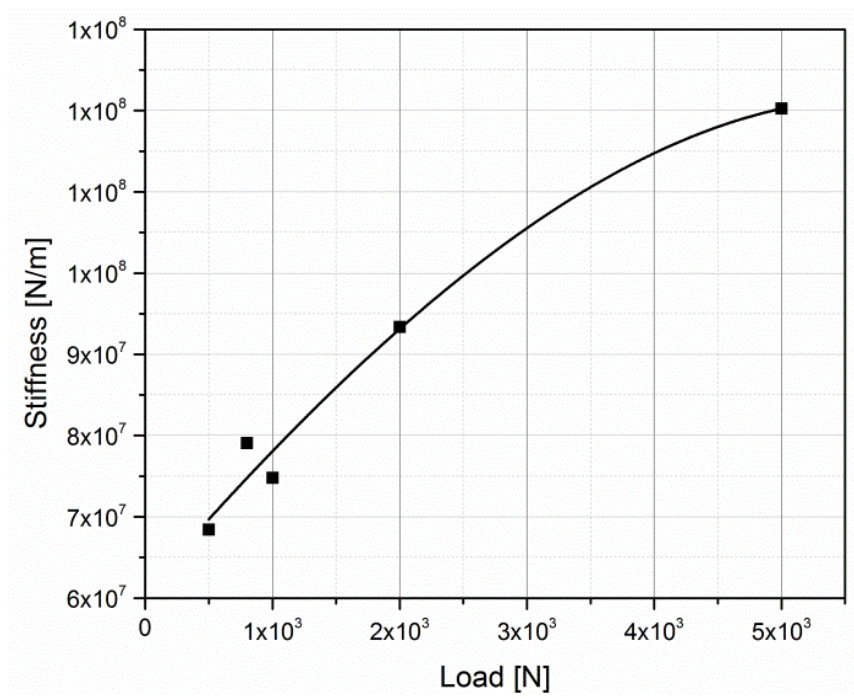

b)

Fig. 7 Radial deformation (a) and radial stiffness (b) as a function of external load

The obtained values were included in equation (7), which represents a differential equation for bearing vibrations with one degree of freedom. These mathematical relations were solved by the Matlab software package and all results obtained for accelerations for all analysed cases of load are shown in Figure 8. For a clear presentation of the influence of the amplitude on acceleration, a comparative overview of the calculated accelerations as well as for the displacements and velocities is given in Figure 9. 


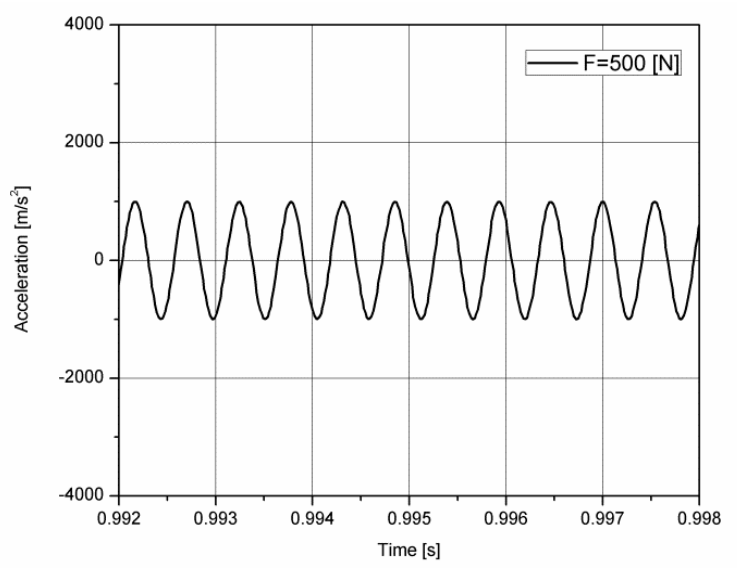

a)

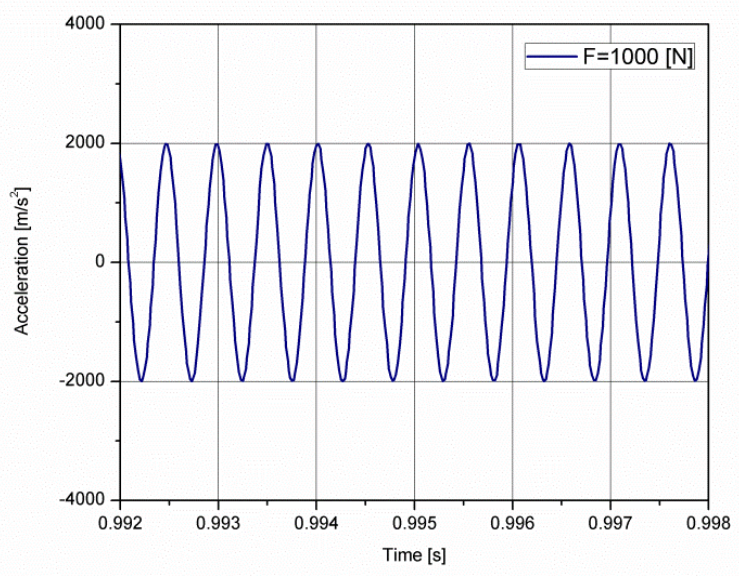

c)

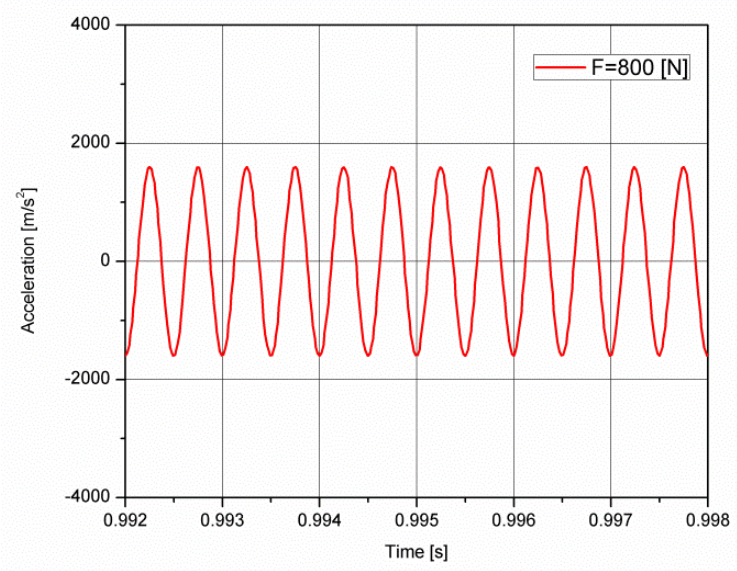

b)

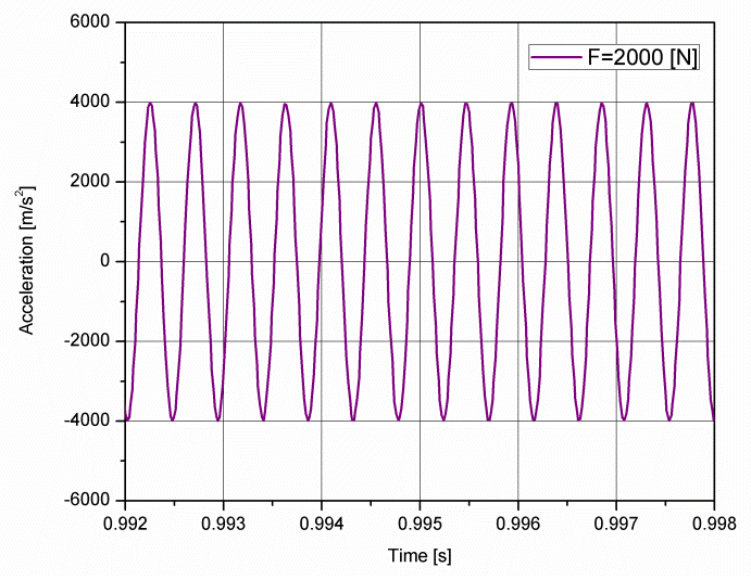

d)

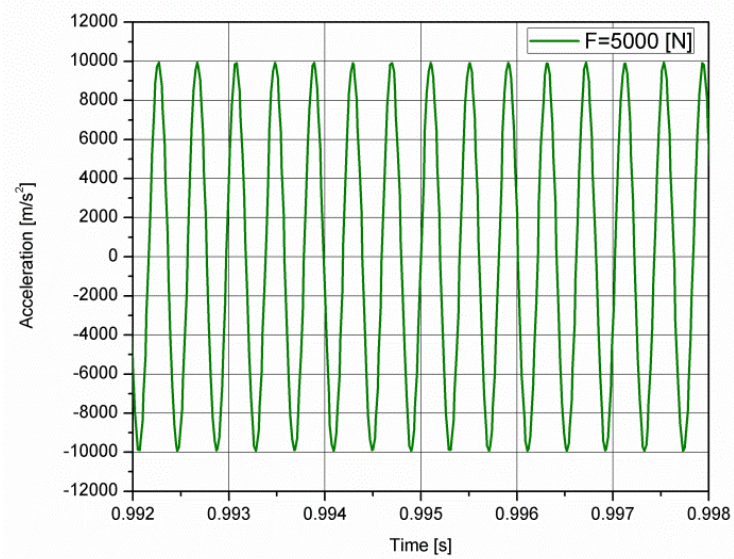

e)

Fig. 8 Vibrations for 6206 ball bearing for different external loads: a) $500 \mathrm{~N}$, b) $800 \mathrm{~N}$, c) $1000 \mathrm{~N}, \mathrm{~d}) 2000 \mathrm{~N}$ and e) $5000 \mathrm{~N}$ 


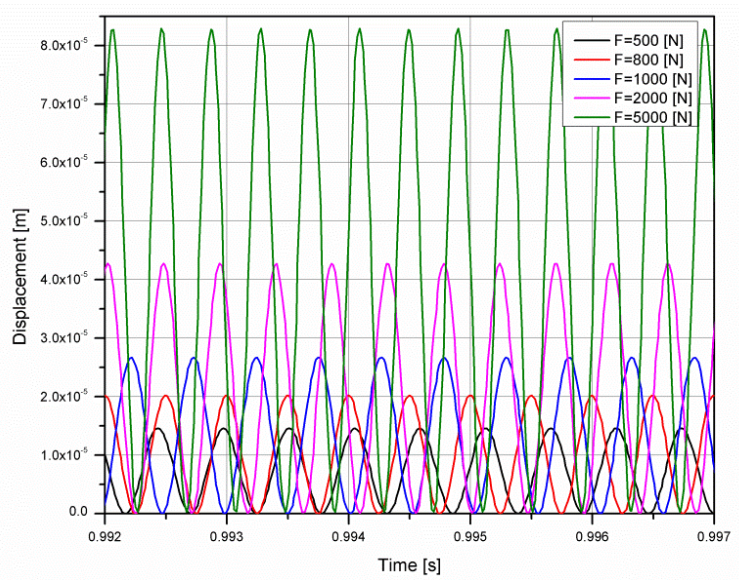

a)

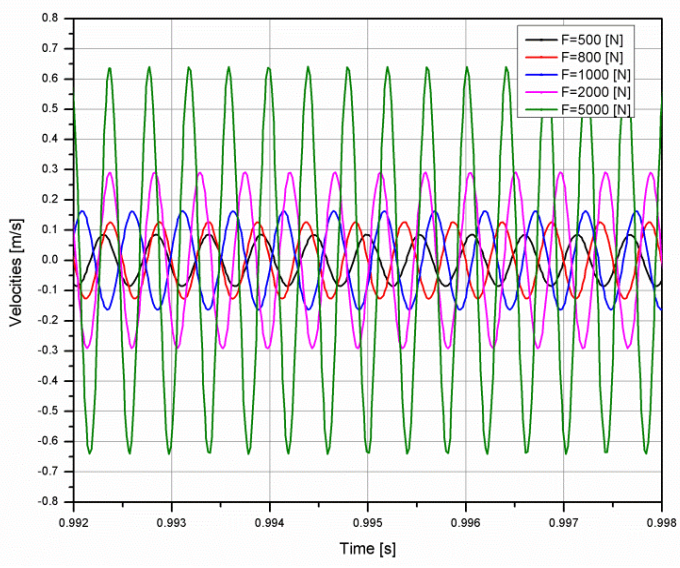

b)

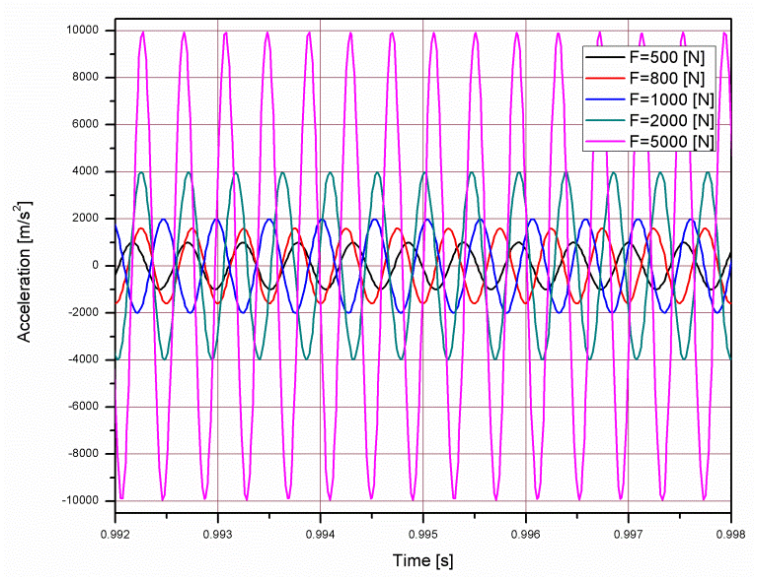

c)

Fig. 9 A comparative overview of results for different loads acting on 6206 bearing: a) displacements, b) velocities and c) accelerations

\section{Experimental verification}

An experimental study was performed with the aim to verify the analytical/numerical methodology developed for analysing the dynamic behaviour of the bearing. In the experiment, a test rig was installed for the dynamic testing of ball bearings, presented in Figure 10.

The device used for the dynamic testing of ball bearings enabled measurements and analysis of relative vibrations (displacement) between the outer race of the bearing and the rotor and absolute vibrations of the outer race of the bearing. To measure the absolute vibrations of the outer race of the bearing it is possible to use measurements of vibration speed or vibration acceleration. For the purpose of the analysis of vibrations of radial bearings, vibrations measurement sensors were placed in such a way that they measured vibrations in the vertical radial direction, which corresponds to the direction of the external load.

During the experiment, only the intensity of the external radial load was varied, i.e. it was gradually increased until the static load bearing capacity i.e. the maximum permissible load on the structure was reached (when the bearing was tested, the load was in the range from $500 \mathrm{~N}$ to $5000 \mathrm{~N}$ ). 
The test rig for the dynamic testing of ball bearings consists of a shaft (1) on which the bearings to be tested are mounted. The shaft is driven by an electromotor (2) by means of belt transmission. All measurements were performed at a constant angular velocity of the shaft rotation $n=1476 \mathrm{~min}^{-1}$, i.e., $\omega=154.5 \mathrm{~s}^{-1}$.

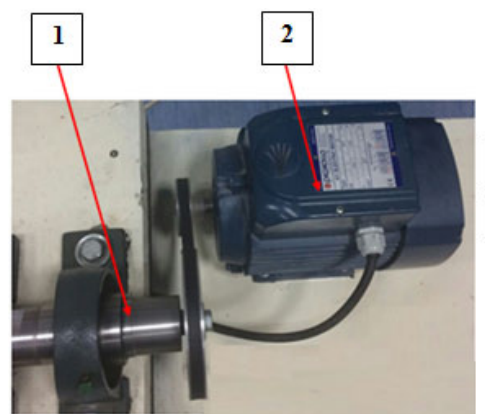

a)

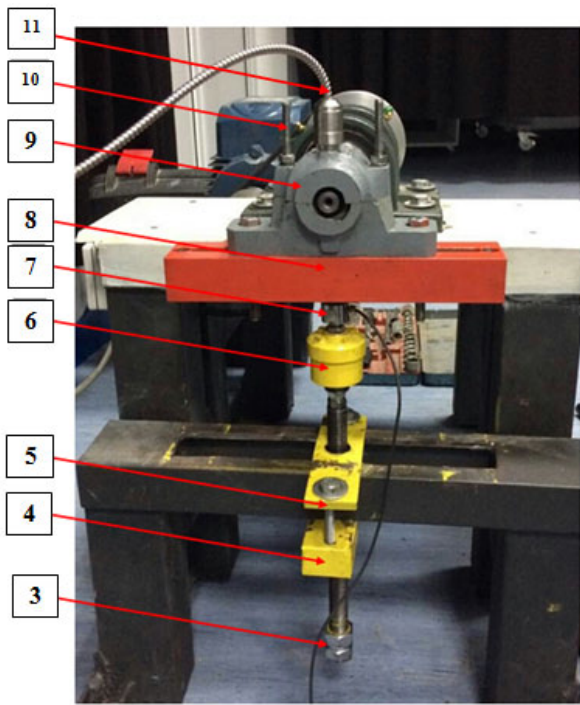

b)

Fig. 10 Experimental set-up for dynamic testing of ball bearings: a) top view and b) side view

The radial load required for the testing of ball bearings was obtained from a threaded spindle (3) and a plate (4), with a threaded hole in the middle. Plate (4) together with plate (5) and their screws serve for the horizontal adjustment of the position of the assembly for imposing an external load. The axial force is transferred forward through an assembly (6) and a sensor (7) to a rigid support (8). The assembly (6) ensures that only the axial movement of the threaded spindle is transmitted to the sensor (7), not its rotation. The force is further transmitted from the support to the bearing cover (9) by two screws with nuts (10), where it is distributed to the outer ring of the bearing [20].

A data acquisition system A2 logger was used for processing and presenting the results. The dynamic behaviour of the bearing was monitored using an accelerometer and a sensor for measuring the shaft displacement (11). The measured results were transferred to the data acquisition device and displayed on the monitor. Programs such as LabVIEW, Matlab and Origin Pro were used as software support to this experiment [3].

The data and measurement results were saved to a disk using data acquisition systems. Further data processing including conversion of electrical signal values (electric tension or amperage) into corresponding physical values was performed in this study by applying the Matlab program package [3].

The value of the specific frequency of the outer ring of the tested bearings $\left(f_{0}\right)$ was obtained from the following equation:

$$
f_{0}=\frac{z}{2} \omega\left(1-\frac{d_{b}}{d_{c}} \cos \alpha\right)
$$

where

$$
\begin{aligned}
& \omega-\text { the angular speed of the rotor, } \\
& z-\text { the total number of the rolling elements (balls), }
\end{aligned}
$$


$d_{b}$ - the diameter of the rolling elements, SKF catalogue value,

$d_{c}$ - the dividing axis of the bearing cage, SKF catalogue value,

$d_{b} / d_{c}=0.207$

$\alpha$ - the contact angle of the bearing.

The radial ball bearing subjected to load or only to radial load that was selected in the experiment and the numerical simulations has the value of the contact angle equal to zero, i.e. $\alpha=0$ [20].

In the frequency diagram of the absolute bearing vibration, characteristic peaks that approximately correspond to the calculation values of the specific frequency of the outer ring of the tested bearings $\left(f_{0}\right)$ are looked for. After that, the values of the vibration amplitude of the bearings on the specific frequencies of the outer ring of the bearing are read.

The results of the measured values of the acceleration amplitudes for the SKF 6206 bearing and for the external load of $1000 \mathrm{~N}$ are shown in Figure $11[3,20]$. The obtained difference between the measured vibration amplitude (Figure 11) and the calculated one for the same bearing type and the same loading conditions (Figure 8c) is in the range of about $2.5 \%$, which proves that there is excellent agreement between the used methods. The calculated and the measured amplitudes and their comparison for all load cases are presented in Table 1. It has been concluded that the newly developed procedure for the analysis of ball bearing vibrations is verified.

Table 1 Calculated and measured amplitudes in $\mathrm{m} / \mathrm{s}^{2}$ for all load cases

\begin{tabular}{|c|c|c|c|c|c|}
\hline $\mathrm{F} / \mathrm{N}$ & 500 & 800 & 1000 & 2000 & 5000 \\
\hline Measured & 750 & 1810 & 1920 & 3820 & 9730 \\
\hline $\begin{array}{c}\text { According to the } \\
\text { developed } \\
\text { methodology }\end{array}$ & 1000 & 1750 & 2000 & 4000 & 10000 \\
\hline
\end{tabular}

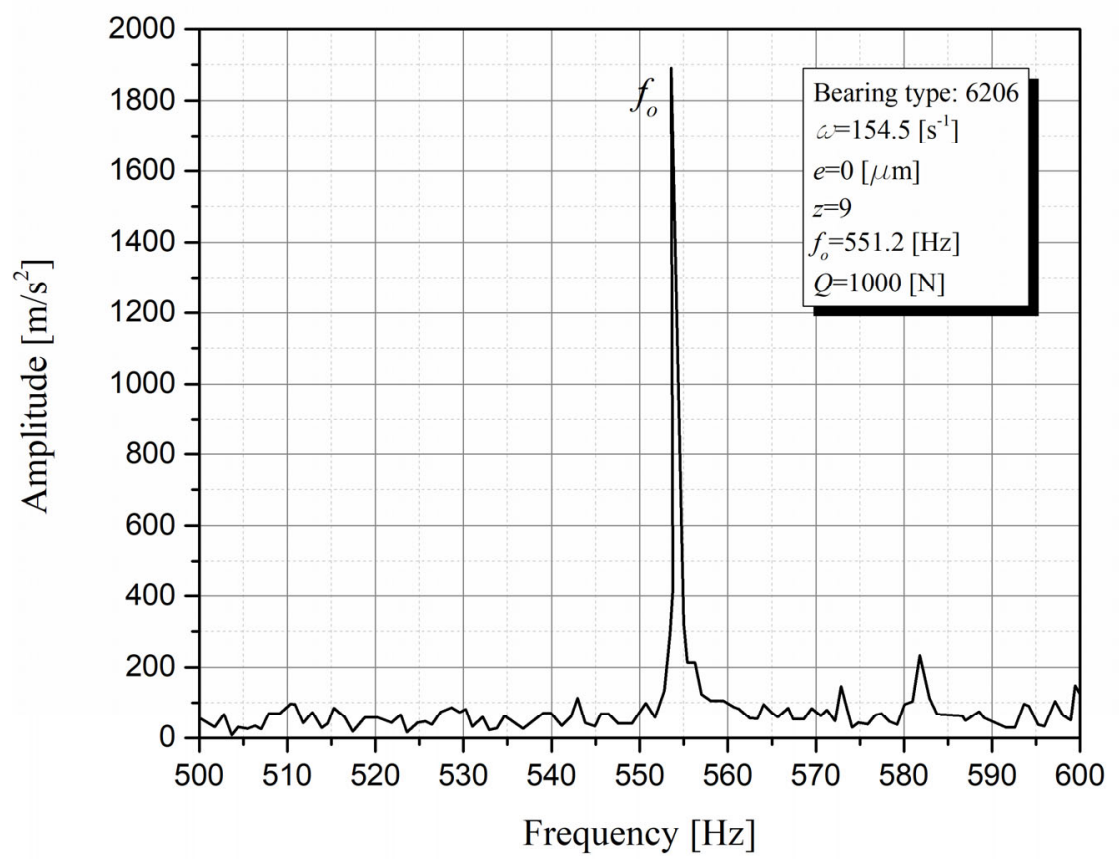

Fig. 11 Results for measured values of acceleration amplitudes for SKF 6206 bearing and for load of 1000 N, $[3,20]$ 


\section{Conclusions}

This paper presents results of the analytical/numerical and experimental study on dynamic behaviour of radial ball bearings. The effects of different levels of load on the dynamic behaviour of ball bearings were analysed. Change in deformation and stiffness as one of the main causes of vibrations in ball bearings was analysed. The developed model of the bearing dynamic behaviour was verified by comparing the model with the results of the experimental study. The obtained results showed that the vibration amplitude increases with a similar multiple factor as load increases.

Based on the analysis of the obtained results it was concluded that a high level of superposition exists between the relations obtained with the new methodology and the experimental measurements, with respect to the external load influence on the ball bearing dynamics. The agreement between the experimental and the numerical results is about $2.5 \%$. Also, a strong effect of the intensity of radial load on the change in stiffness was observed.

The described ball bearing model, developed by using the finite element method, was initially aimed at the precise modelling of the geometries of the bearing elements in contact. It is expected that this model contributes significantly to future investigations into the ball bearing dynamics when different deviations in geometry are present on roller races. That is undoubtedly of high interest in multi-scale research [18]. Another main contribution is the methodology which has been developed and described in this paper. It is uniform and suitable for the analysis of effects of different influential parameters on the bearing dynamics, e.g. angular velocity and rotation, and could have a wide application in the future.

\section{Acknowledgments}

We show our gratitude to the Factory manufacturing rolling bearings and Cardan shafts FKL Temerin, Serbia, for providing data and samples used in this study.

Parts of this investigation were supported by the Ministry of Education, Science and Technology of the Republic of Serbia through Grant TR 35029 and Grant OI 174001.

\section{REFERENCES}

[1] Harsha, S.P. Nonlinear dynamic response of a balanced rotor supported by rolling element bearings due to radial internal clearance effect, Mechanism and Machine Theory 2006, 41 (6), 688-706. https://doi.org/10.1016/j.mechmachtheory.2005.09.003

[2] Changqing, B.; Qingyu, X. Dynamic model of ball bearings with internal clearance and waviness, Journal of Sound and Vibration, 2006, 294, 23-48. https://doi.org/10.1016/j.jsv.2005.10.005

[3] Tomović, R.; Miltenović, V.; Banić, M.; Miltenović, A. Vibration response of rigid rotor in unloaded rolling element bearing, International Journal of Mechanical Sciences 2010, 52 (9), 1176-1185. https://doi.org/10.1016/j.ijmecsci.2010.05.003

[4] Harris, T.A.; Kotzalas, M.N. Rolling Bearing Analysis 2007, Taylor\&Franciz Group, SAD

[5] Liu, J.; Shao, Y. Vibration modelling of nonuniform surface waviness in a lubricated roller bearing, Journal of Vibration and Control 2015, 23 (7), 1115-1132. https://doi.org/10.1177/1077546315589675

[6] Jang, G.H.; Jeong, S.W. Analysis of a ball bearing with waviness considering the centrifugal force and gyroscopic moment of the ball, ASME Journal of Tribology 2003, 125 (3), 487-498. https://doi.org/10.1115/1.1538618

[7] Adamczak, S.; Zmarzły, P. Influence of raceway waviness on the level of vibration in rolling-element bearings, Bulletin of the polish academy of sciences technical sciences 2017, 65 (4), 541-551. https://doi.org/10.1515/bpasts-2017-0059

[8] Aktfluk, N. The effect of waviness on vibrations associated with ball bearings, ASME Journal of Tribology 1999, 121(4), 667-677. https://doi.org/10.1115/1.2834121

[9] Ristivojević, M.; Mitrović, R. Load distribution - gear pairs and rolling bearings, Monograph 2002. ISBN 86-7558-112-2 
[10] Sharma, A; Upadhyay, N.; Kumar Kankar, P.; Muniyappa, A. Nonlinear dynamic investigations on rolling element bearings: A review, Advances in Mechanical Engineering 2018, 10(3), 1-15. https://doi.org/10.1177/1687814018764148

[11] Sunnersjo, C. S. Varying compliance vibrations of rolling bearings, Journal of Sound and Vibration 1978, 58 (3), 363-373. https://doi.org/10.1016/S0022-460X(78)80044-3

[12] Marsh, E. R.; Yantek, D. S. Experimental measurement of precision bearing dynamic stiffness, Journal of Sound and Vibration 1997, 202 (1), 55-66. https://doi.org/10.1006/jsvi.1996.0793

[13] Zhang, X.; Yin, Z.; Gao, G.; Li, Z. Determination of stiffness coefficients of hydrodynamic waterlubricated plain journal bearings, Tribology International 2015, 85, 37-47. https://doi.org/10.1016/j.triboint.2014.12.019

[14] Mitrović, R.; Soldat, N.; Atanasovska, I. Dynamic Behavior of Radial Ball Bearing due to the Periodic Variable Stiffness, Machine Design 2015, 7 (1), 1-4. ISSN 1821-1259

[15] Atanasovska, I.; Mitrović, R.; Stefanović, S.; Soldat, N.; Mišković, Ž. Calculation of Radial Stiffness for Single-row Ball Bearing with Finite Element Analysis, Journal Machine Design 2014, 6 (3), 85-90. ISSN 1821-1259

[16] Atanasovska, I. The Mathematical Phenomenological Mapping in Nonlinear Dynamics of Spur Gear Pair and Radial Ball Bearing due to the Variable Stiffness, International Journal of Non-linear Mechanics 2015, 73, 114-120. https://doi.org/10.1016/j.ijnonlinmec.2014.11.015

[17] Atanasovska, I.; Mitrović, R.; Soldat, N. Developing the finite element model for dynamic analysis of radial ball bearing, 5th International Congress of Serbian Society of Mechanics 2015, 1-6. ISBN 978-867892-715-7, COBISS.SR-ID 296997639

[18] Atanasovska, I.; Mitrović, R.; Soldat, N. Influence of Load Distribution in Ball Bearings with Defects on the Dynamic Behavior of Gear Transmissions Systems, Proceedings of International Conference on GEARS 2015, 2015, 1065-1068. ISSN 0083-5560, ISBN 978-3-18-092255-3

[19] Liu, J.; Shao, Y.; Zhu, W.D. A New Model for the Relationship Between Vibration Characteristics Caused by the Time-Varying Contact Stiffness of a Deep Groove Ball Bearing and Defect Sizes, ASME Journal of Tribology 2015, 137 (3), 031101/1- 031101/15. https://doi.org/10.1115/1.4029461

[20] Tomović, R. Research of rolling bearings construction parameters impact to the condition of their labouring correctness, Doctoral dissertation 2009, University of Niš, Faculty of Mechanical Engineering

Submitted: $\quad 01.12 .2018$

Accepted: $\quad$ 26.7.2019
Nataša Soldat

Full Prof. Radivoje Mitrović

University of Belgrade - Faculty of

Mechanical Engineering, Kraljice Marije 16, 11000 Belgrade, Serbia

Ivana Atanasovska

Mathematical institute of SASA, Kneza

Mihaila 36, 11000 Belgrade, Serbia

Assoc. Prof. Radoslav Tomović

Faculty of Mechanical Engineering,

University of Montenegro, Džordža

Vašingtona bb, 81000 Podgorica,

Montenegro 